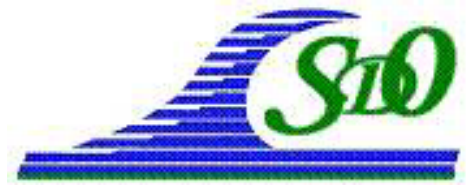

XI ìmes Journées Nationales Génie Côtier - Génie Civil

Les Sables d'Olonne, 22-25 juin 2010

DOI:10.5150/jngcgc.2010.004-B C Editions Paralia CFL

disponible en ligne - http://www.paralia.fr - available online

\title{
Validation numérique d'un modèle double-couche de type Boussinesq pour des ondes fortement non-linéaires et dispersives
}

\author{
Michel BENOIT ${ }^{1}$, Florent CHAZEL ${ }^{2}$
}

1. Laboratoire d'Hydraulique Saint-Venant, Université Paris-Est, (unité de recherche commune EDF R\&D, CETMEF, Ecole des Ponts ParisTech), 6 quai Watier, 78400 Chatou, France.

michel.benoit@edf.fr

2. Université de Toulouse, UPS/ Institut National des Sciences Appliquées, IMT, CNRS UMR 5219,

135 avenue de Rangueil, 31077 Toulouse cedex 4, France.

florent.chazel@insa-toulouse.fr

\section{Résumé :}

Un modèle original, de type Boussinesq, basé sur une approche double-couche a récemment été proposé par les auteurs (CHAZEL et al., 2009), permettant de modéliser des vagues fortement non-linéaires et dispersives en zone proche côtière et côtière. Ce modèle présente notamment d'excellentes propriétés linéaires jusqu'à $k h=10$ (où $k$ est le nombre d'onde et $h$ la hauteur d'eau) tant pour les profils verticaux de vitesses orbitales que pour le shoaling. Après un bref résumé de la construction mathématique du modèle, nous présentons sa mise en œuvre numérique en une dimension d'espace horizontale. Le modèle est ensuite appliqué à deux cas expérimentaux, afin d'estimer son domaine de validité non-linéaire pour des profondeurs d'eau intermédiaires à grandes. En premier lieu, nous considérons les expériences classiques de DINGEMANS (1994) étudiant la propagation de vagues régulières au-dessus d'une barre trapézoïdale immergée. Les résultats numériques obtenus démontrent une excellente capacité du modèle à reproduire les effets de shoaling, les interactions fortement non-linéaires, ainsi que la génération puis la propagation d'harmoniques d'ordre élevé après la barre. Ensuite, nous mettons en exergue la capacité du modèle à propager des vagues irrégulières non-déferlantes, en simulant une des expériences en canal de BECQGIRARD et al. (1999) sur une bathymétrie plus complexe, comportant également un haut fond. Dans ce deuxième cas, les résultats du modèle sont également en très bon accord avec les mesures expérimentales.

\section{Mots-clés :}

Vagues non-linéaires - Houle côtière - Modèle de type Boussinesq - Technique double-couche - Approximants de Padé - Vagues dispersives 


\begin{abstract}
:
A new Boussinesq-type model, based on a two-layer approach, has recently been proposed by the authors (CHAZEL et al., 2009), allowing to simulate highly nonlinear and dispersive waves in the nearshore and coastal zone. In particular this model exhibits excellent linear properties up to $k h=10$ (where $k$ is the wave-number and $h$ the water depth) both for the vertical profile of orbital velocities and for the shoaling coefficient. After a brief summary of the mathematical derivation of the model, we present its numerical implementation for one-dimensional cases. The model is then applied to two experimental cases in order to study its range of applicability in the nonlinear regime for intermediate to large water depths. We first consider the classical experiments by DINGEMANS (1994) addressing the propagation of regular waves over a trapezoidal submerged bar. The results of the numerical model show its excellent performance in reproducing shoaling effects, strongly nonlinear interactions, as well as the generation and the release of higher order harmonics after the bar. Then we illustrate the capability of the model to propagate irregular non-breaking waves, by simulating one of the flume experiments by BECQ-GIRARD et al. (1999) with a more complex bottom profile also including an underwater bar. For this second case the model's results are also in very good agreement with experimental measurements.
\end{abstract}

\title{
Keywords :
}

Nonlinear waves - Coastal waves -Boussinesq-type model - Two-layer technique Padé approximants - Dispersive waves

\section{Introduction}

Durant les deux dernières décennies, les modèles de type Boussinesq se sont imposés comme un outil performant et couramment utilisé par la communauté scientifique en ingénierie côtière. A l'origine, les modèles de ce type se basaient sur deux hypothèses fondamentales, celle de faible non-linéarité et celle de faible dispersion des ondes de surface, ce qui leur conférait un domaine de validité limité aux eaux peu profondes et aux vagues de faibles amplitude (voir par exemple PEREGRINE, 1967). De nombreux efforts ont depuis porté sur l'extension du domaine de validité linéaire et non-linéaire de ces modèles. Les travaux de NWOGU (1993) et WEI et al. (1995) tout d'abord, puis ceux de GOBBI et al. (2000), AGNON et al. (1999) et MADSEN et al. (2002) notamment, ont ainsi permis de s'affranchir de l'hypothèse de faible non-linéarité, puis de celle de faible dispersion, permettant ainsi de simuler correctement des ondes fortement non-linéaires jusqu'en eaux profondes, mais au prix d'un accroissement conséquent de la complexité numérique des modèles développés, liée à l'utilisation de dérivées spatiales d'ordre élevé (i.e. supérieures à 3).

Guidés par la volonté d'obtenir un modèle applicable sur des domaines côtiers complexes et précis jusqu'en eaux profondes, mais avec une complexité numérique moindre que les modèles mentionnés ci-dessus, nous avons récemment proposé un 
nouveau modèle de type Boussinesq basé sur une approche double-couche (CHAZEL et al., 2009). Ce modèle est présenté brièvement dans la partie 2 de cet article, et sa mise en œuvre numérique est exposée en partie 3. Nous illustrons dans la partie 4 les capacités du modèle sur deux cas de validation : les expériences en canal à vagues de DINGEMANS (1994) avec un haut-fond, et un essai de BECQ-GIRARD et al. (1999) réalisé également en canal à vagues, mais en conditions de vagues irrégulières.

\section{Formulation mathématique du modèle}

\subsection{Présentation générale du modèle}

En s'appuyant sur les hypothèses de pente douce du fond marin et d'irrotationnalité de l'écoulement, et en négligeant les effets visqueux, le problème peut s'écrire sous forme potentielle, diminuant ainsi le nombre total d'inconnues. La construction du modèle combine trois idées : premièrement, l'approche double-couche proposée par LYNETT \& LIU (2004), (voir figure 1 pour une définition du domaine de calcul), permettant de diminuer l'ordre des dérivées du problème en séparant artificiellement le fluide en deux couches (de même densité). La deuxième idée consiste à formuler le problème en fonction d'un opérateur de Dirichlet-Neumann exprimé, non pas à la surface libre (i.e. en $z=\eta(x, y)$ ), mais à la surface au repos (i.e. en $z=0$ ), afin de tirer parti de son caractère statique. Enfin, la dernière idée repose sur la méthode proposée par AGNON et al. (1999) et MADSEN et al. (2002), qui vise à rechercher des solutions à l'équation de Laplace sous la forme de séries de Taylor infinies, puis à les tronquer à un certain ordre afin d'utiliser des approximants de Padé. Le modèle final est ainsi composé de quatre équations que le domaine spatial soit unidimensionnel et bidimensionnel, et n'inclut que dérivées spatiales d'ordre deux au maximum, ce qui représente un avantage conséquent vis-à-vis des modèles de Boussinesq précédemment cités.

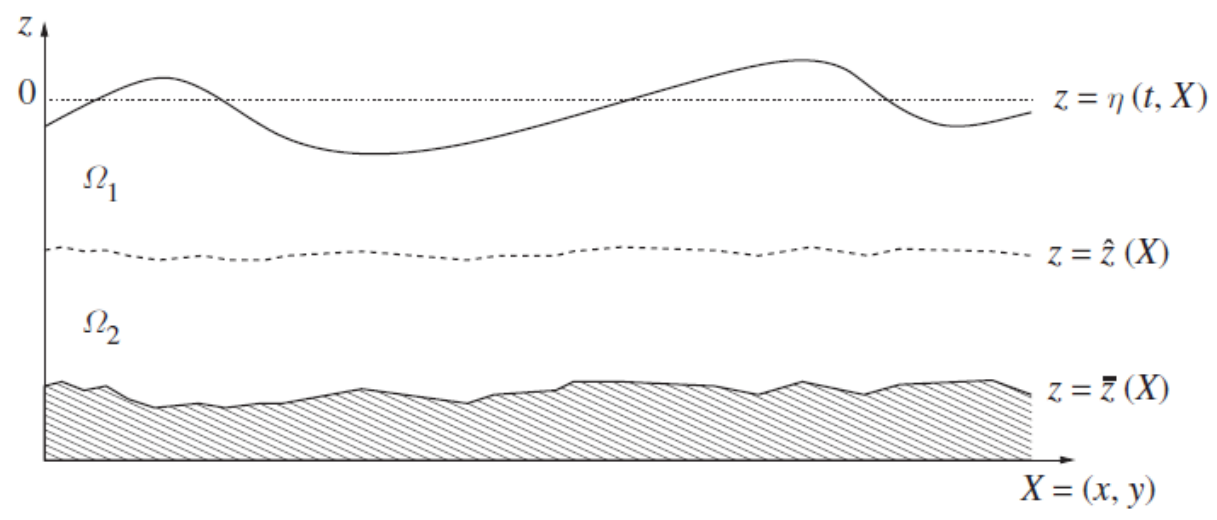

Figure 1. Représentation du domaine fluide et notations principales. 


\subsection{Formulation mathématique du modèle}

Après développements (voir CHAZEL et al., 2009), le modèle s'écrit au final :

$$
\left.\begin{array}{c}
\partial_{t}{\widetilde{\phi_{1}}}+\frac{1}{2} \mid \nabla{\widetilde{\phi_{1}}}^{2}-\frac{1}{2}{\widetilde{w_{1}}}^{2}\left(1+|\nabla \eta|^{2}\right)+g \eta=0, \\
\partial_{t} \eta+\nabla \eta \cdot \nabla \widetilde{\phi}_{1}-\widetilde{w}_{1}\left(1+|\nabla \eta|^{2}\right)=0, \\
\left(1-\frac{\eta^{2}}{2} \Delta+\left(\eta-\frac{\eta^{3}}{6} \Delta\right) \mathcal{G}_{0}^{\mathrm{app}}[h]\right) \phi_{0}=\widetilde{\phi}_{1}, \\
\widetilde{w}_{1}=\left(-\eta \Delta+\left(1-\frac{\eta^{2}}{2} \Delta\right) \mathcal{G}_{0}^{\mathrm{app}}[h]\right) \phi_{0},
\end{array}\right\}
$$

où $\eta(x, y)$ désigne l'élévation de la surface libre, $\widetilde{\phi}_{1}(x, y)$ le potentiel des vitesses à la surface libre, et $\widetilde{w}_{1}(x, y)$ la vitesse verticale à la surface libre. L'opérateur $\mathcal{G}_{0}^{\text {app }}[h]$ désigne quant à lui une approximation de l'opérateur de Dirichlet-Neumann exprimé à la surface au repos, et ne fait intervenir que des dérivées d'ordre deux au maximum. Cet opérateur contient la condition à la limite sur le fond qui est de type "glissement» (condition de type Neumann sur le potentiel). L'expression complète de l'opérateur $\mathcal{G}_{0}^{\text {app }}[h]$ est donnée dans CHAZEL et al. (2009). Les dérivées spatiales apparaissant dans la formulation ci-dessus (opérateurs $\nabla$ et $\Delta$ ) sont des dérivées par rapport aux variables d'espace horizontales $(x, y)$.

\subsection{Analyse linéaire du modèle}

Une analyse linéaire du modèle est menée afin d'évaluer les propriétés dispersives du modèle dans sa version linéarisée, en particulier la vitesse de phase, les profils verticaux de vitesse horizontale ainsi que le coefficient de shoaling. Celles-ci sont comparées aux résultats issus de la théorie linéaire de Stokes. Les résultats pour la célérité de phase à fond plat sont présentés à titre d'exemple sur la figure 2 .

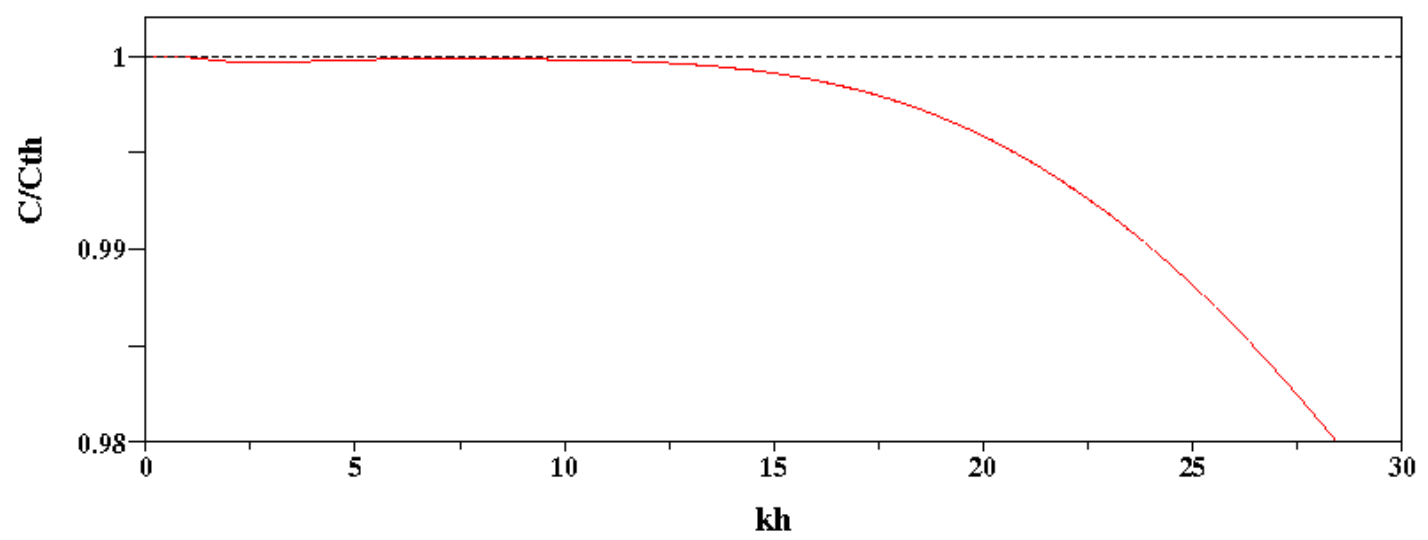

Figure 2. Rapport entre la vitesse de phase du modèle linéarisé et la vitesse de phase donnée par la théorie de Stokes à l'ordre 1, en fonction de $\mathrm{kh}$. 
En tirant parti de la position de l'interface entre les deux couches (cf. figure 1), il est possible d'optimiser les propriétés dispersives du modèle, qui s'avèrent au final excellentes jusqu'en eaux profondes (i.e. pour des valeurs de $k h$ supérieures à 10) et équivalentes à celles obtenues dans MADSEN et al. (2002), pour une complexité mathématique et numérique nettement moindre.

\section{Mise en œuvre numérique du modèle en une dimension d'espace horizontale}

Le modèle mathématique développé a été simulé numériquement en une seule dimension d'espace horizontale (modèle 2DV) au travers d'un code de calcul en différences finies développé sous SCILAB. Les dérivées premières et secondes sont approchées par des formules d'ordre 4 calculées sur des stencils de 5 points. Les conditions aux limites sur les inconnues sont imposées de la manière suivante : les coefficients correspondant aux points du stencil sortant du domaine de calcul sont réfléchis de manière paire pour une condition de type Neumann, et impaire pour une condition de type Dirichlet homogène. Cette méthode permet en particulier de conserver une structure très régulière dans les matrices de discrétisation.

Afin de reproduire numériquement le comportement d'un bassin à houle, des zones de relaxation sont mises en place en entrée et en sortie du domaine de calcul (cf. BINGHAM \& AGNON, 2005) afin d'assurer la génération et l'absorption des vagues. Sur ces zones de relaxation et à chaque pas de temps, la valeur finale de chaque inconnue est calculée par combinaison convexe de la solution numérique et de la solution que l'on cherche à imposer. Cette dernière pourra par exemple correspondre aux expressions linéaires du potentiel et de la surface libre issues du développement de Stokes au premier ordre, ou bien être identiquement nulle dans le cas d'une zone d'absorption. Dans les deux cas, des conditions de type Neumann sont imposées en conditions aux limites sur le potentiel et la surface libre au niveau des frontières latérales. Enfin, l'intégration en temps du modèle est effectuée à l'aide d'un schéma de Runge-Kutta explicite à 4 pas, d'ordre 4 , avec un pas de temps constant.

\section{Validation du modèle}

\subsection{Propagation de vagues régulières au-dessus d'une barre immergée}

Nous examinons ici la capacité du modèle à décrire la propagation et les interactions entre ondes très dispersives. Dans ce but, nous nous intéressons à la propagation de vagues régulières au-dessus d'une barre trapézoïdale immergée, en suivant le dispositif utilisé par DINGEMANS (1994) et décrit sur la figure 3. Des vagues régulières sont générées en entrée de domaine (limite gauche) et se propagent en direction de la barre. Au passage de celle-ci, la diminution de la profondeur entraine un raidissement et une augmentation de l'amplitude des vagues. Les interactions vague-vague non-linéaires génèrent des composantes harmoniques d'ordres élevés, liées au mode fondamental et 
donnant des profils de vagues asymétriques (par rapport à un plan horizontal et vertical). Ces harmoniques sont ensuite libérées sur la partie plate du profil, puis lorsque la profondeur d'eau augmente à nouveau, pour devenir des ondes fortement dispersives se propageant librement au-delà de la barre. Une partie significative de l'énergie des vagues se situe alors dans le domaine $k h>4$, ce qui rend ce cas-test extrêmement exigeant au niveau des propriétés dispersives du modèle testé, ainsi que des propriétés non-linéaires de ce dernier. On présente tout d'abord les résultats du cas $\mathrm{A}(H=2,0 \mathrm{~cm}$; $T=2,02 \mathrm{~s})$ pour lequel les ondes incidentes sont relativement longues $(k h=0,67$ au large de la barre) et de cambrure relativement faible $(k H / 2=0,017$ ou $H / L=0,0053)$.

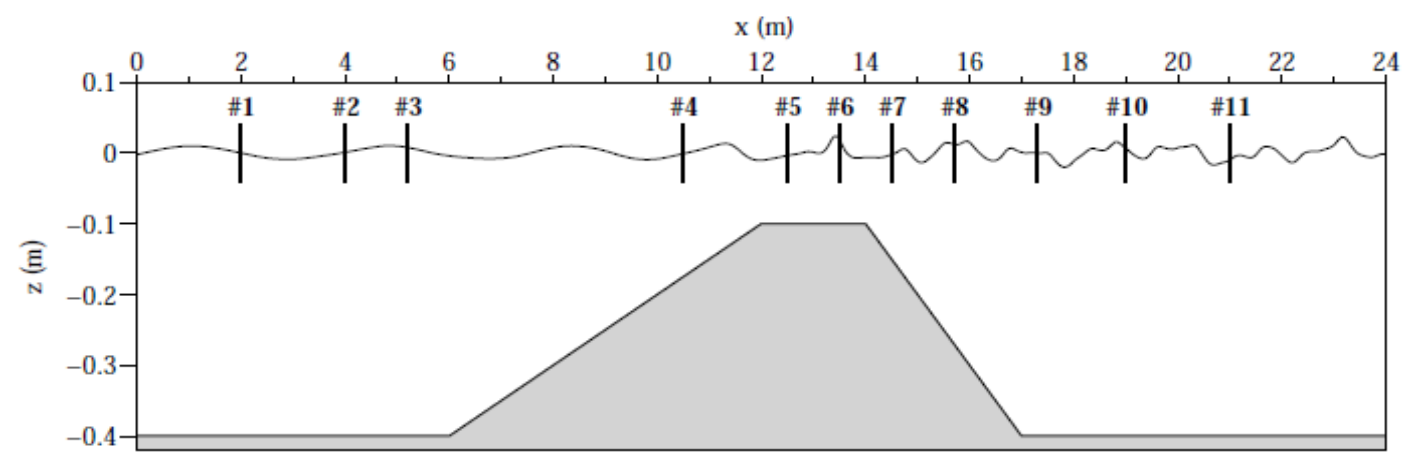

Figure 3. Dispositif expérimental de DINGEMANS (1994) : profil bathymétrique et position des sondes de mesure de vague.

Sur la figure 4, les profils de surface libre simulés pour le cas A sont comparés aux mesures expérimentales sur une durée de 2 périodes de vague incidente sur les 4 dernières sondes. Ces sondes, situées derrière la barre, sont celles en lesquelles les effets non-linéaires et dispersifs se manifestent de la façon la plus marquée. On distingue en effet nettement l'effet de "désintégration" du train d'onde incident par la barre avec la présence d'harmoniques d'ordres supérieurs, qui se propagent avec leur propre célérité (au contrainte de la partie située avant le replat où les harmoniques restent liées au mode fondamental). Cela se traduit par des profils de surface libre très variables d'une sonde à l'autre en fonction des phases relatives des harmoniques ainsi libérés.

Nous avons également simulé le cas $\mathrm{C}$ de cette même série d'expériences, caractérisé par une période de vague incidente plus courte de moitié ( $T=1,01 \mathrm{~s})$ et une hauteur de vague plus importante $(H=4,1 \mathrm{~cm})$. Pour ce cas où les vagues incidentes sont plus courtes $(k h=1,69$ au large de la barre) et présentent une cambrure plus importante $(k H / 2=0,087$ ou $H / L=0,028)$, les résultats du modèle sont également très bons, comme illustré sur la figure 5 pour les profils de surface libre aux quatre dernières sondes de mesure. Sur ce cas C, la part d'énergie transférée du mode fondamental vers les harmoniques supérieurs est cependant plus faible (en relatif) que sur le cas A, ce qui se traduit par un champ de vagues moins irrégulier après le passage de la barre. 

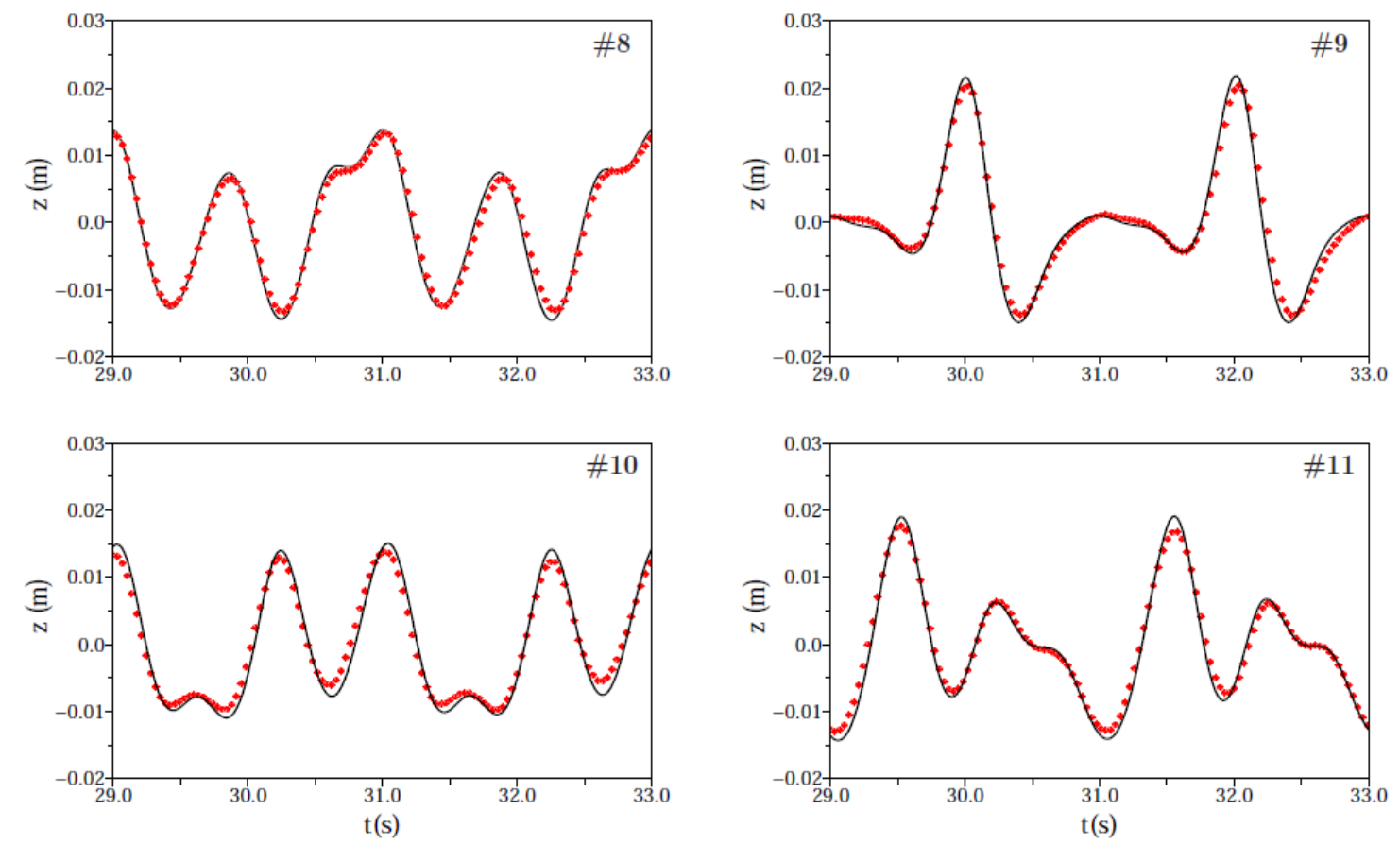

Figure 4. Profils de la surface libre aux dernières sondes pour le cas $A(H=2,0 \mathrm{~cm}$; $T=2,02 \mathrm{~s})$ : résultats du modèle en trait noir, résultats expérimentaux en points rouges.
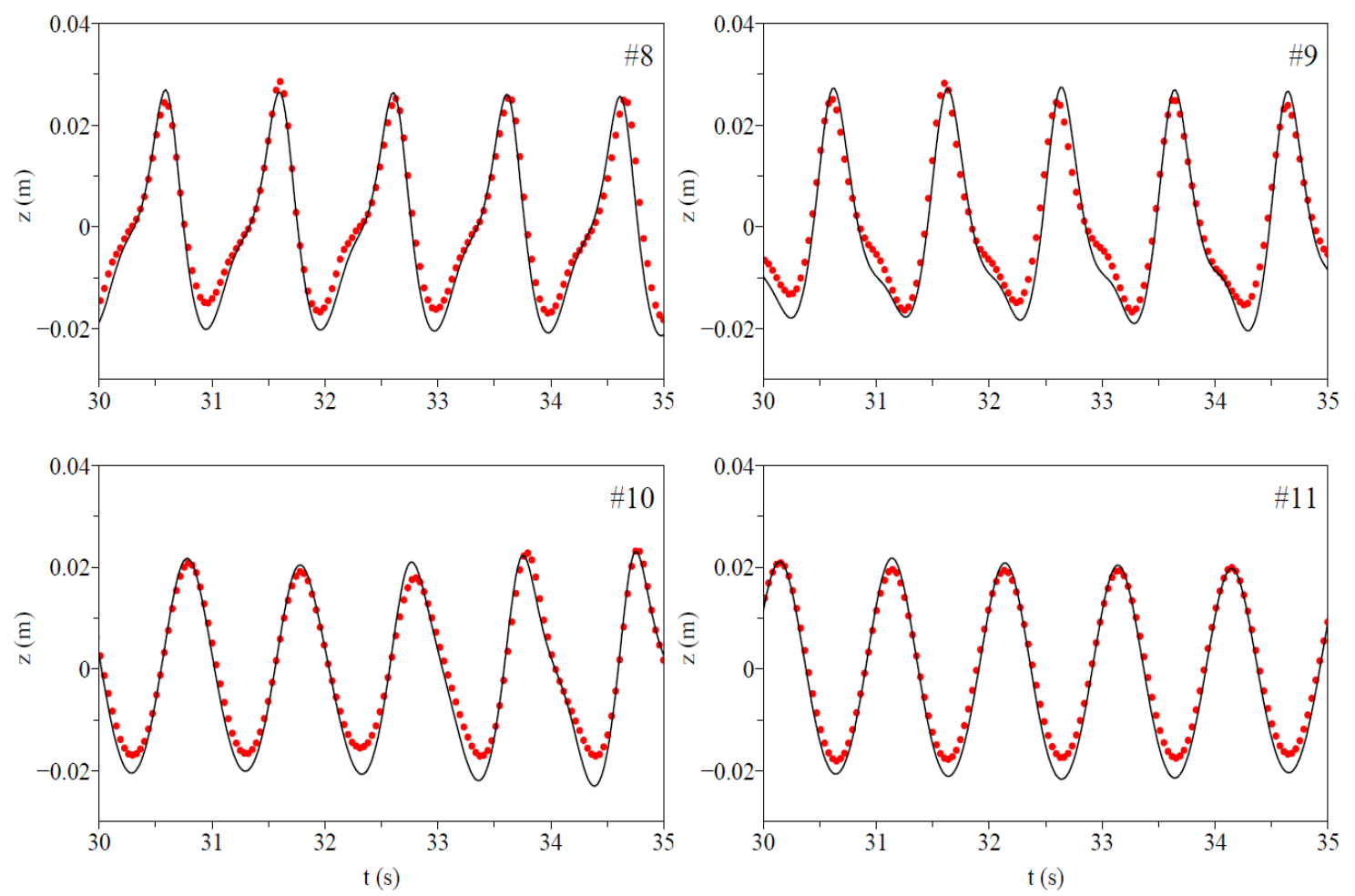

Figure 5. Profils de la surface libre aux dernières sondes pour le cas $C(H=4,1 \mathrm{~cm}$; $T=1,01 \mathrm{~s})$ : résultats du modèle en trait noir, résultats expérimentaux en points rouges. 
Dans les deux cas simulés, les profils de surface libre sont très bien reproduits, pour toutes les sondes situées derrière la barre, que ce soit en terme d'amplitude ou de phase, y compris pour les détails des harmoniques 2 et 3 . L'accord des résultats obtenu ici est bien meilleur que celui que l'on peut attendre de modèles de type Boussinesq étendu tel que ceux de NWOGU (1993) ou WEI et al. (1995) par exemple.

\subsection{Propagation de vagues sur des fonds complexes en faible profondeur d'eau}

Une série d'essais en vagues irrégulières a été réalisée en 1997-1998 à EDF R\&D Chatou dans le cadre de la thèse de F. Becq-Girard (voir BECQ-GIRARD et al., 1999) pour un profil bathymétrique, représenté sur la figure 6 , destiné à mettre en évidence les effets non-linéaires en faible profondeur d'eau. Le canal considéré (canal $n^{\circ} 12$ ) a une longueur de $45 \mathrm{~m}$ pour une largeur de $0,60 \mathrm{~m}$. Il est équipé d'un batteur plan, animé d'un mouvement de type "piston" et piloté par ordinateur. Le profil bathymétrique étudié se compose d'une succession de pentes (plaques métalliques lisses) dont l'inclinaison diminue jusqu'à l'arrivée sur un replat; puis la profondeur augmente à nouveau légèrement, avant de diminuer régulièrement sous la forme d'une pente douce de plage constante (environ 5\%). Les séries temporelles d'élévation de surface libre étaient mesurées en 16 points répartis le long du profil, comme indiqué sur la figure 6 , à l'aide de sondes résistives.

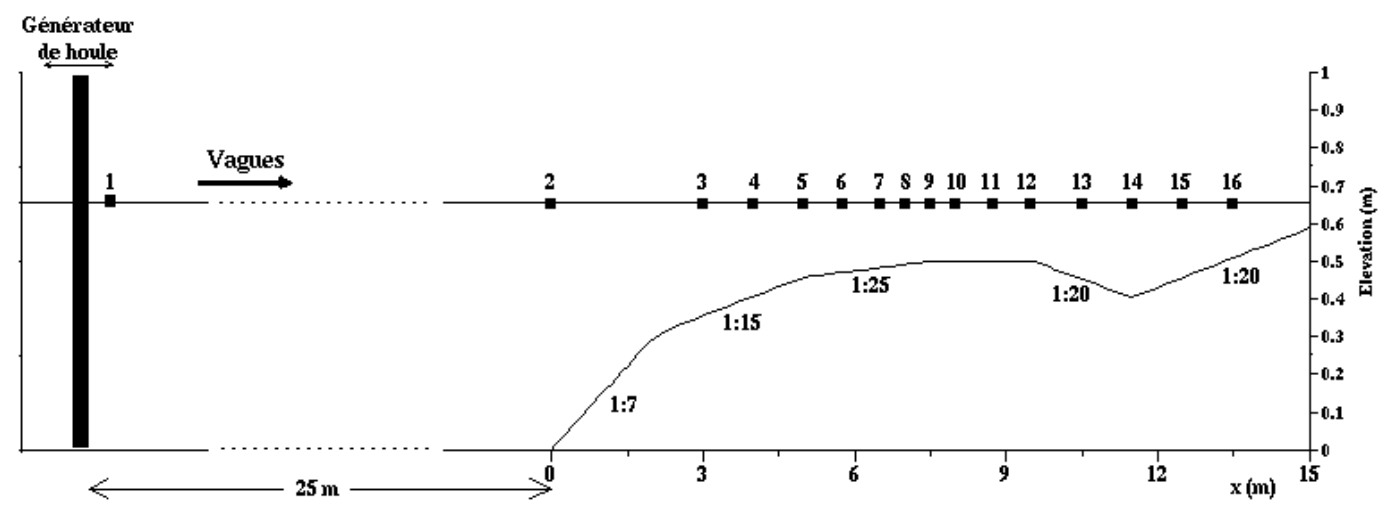

Figure 6. Dispositif expérimental des essais de BECQ-GIRARD et al. (1999) : profil bathymétrique et position des sondes.

Nous considérons ici l'essai 26 de cette série d'expériences, qui correspond à un essai en vague irrégulière et sans déferlement. La profondeur d'eau au large du profil bathymétrique et au niveau du batteur à houle est $h=0,65 \mathrm{~m}$. La hauteur significative des vagues générées est de $H_{m o}=3,4 \mathrm{~cm}$ pour une fréquence de pic de $f_{p}=0,435 \mathrm{~Hz}$ (période de pic $T_{p}=2,3 \mathrm{~s}$ ). Le spectre de vague au batteur est de type JONSWAP avec un facteur d'élancement du pic de 3,3. Les mesures ont été acquises sur une durée de 40 minutes, avec un pas de temps d'échantillonnage de $0,070 \mathrm{~s}$. 
Les profils de surface libre obtenus avec le modèle sont comparés aux mesures expérimentales aux différentes sondes. La figure 7 illustre cette comparaison au niveau de la dernière sonde $\left(\mathrm{n}^{\circ} 16\right)$ sur une durée de $15 \mathrm{~s}$ (soit à peu près 6,5 périodes de pic), où la capacité du modèle à gérer des ondes fortement dispersives peut être mise en exergue. En effet, les interactions fortement non-linéaires entre harmoniques d'ordres élevés aux sondes 4 et 12 perdent en intensité aux sondes 13 à 15 , du fait de l'augmentation de la profondeur d'eau. Ceci a pour effet de libérer ces harmoniques, qui se propagent ainsi librement au niveau de la sonde 16. Le profil obtenu avec le modèle montre un bon accord global avec les résultats expérimentaux : les pics d'amplitude (et les creux) sont bien reproduits, tout comme les légères oscillations du profil liées aux harmoniques d'ordres élevés. Ces différentes harmoniques semblent se propager aux bonnes vitesses, ce qui montre ici encore la très bonne capacité du modèle à reproduire tant les interactions fortement non-linéaires que la propagation d'ondes fortement dispersives. La comparaison des spectres simulés et mesurés est actuellement en cours et sera présentée lors de la conférence et dans la version finale de l'article.

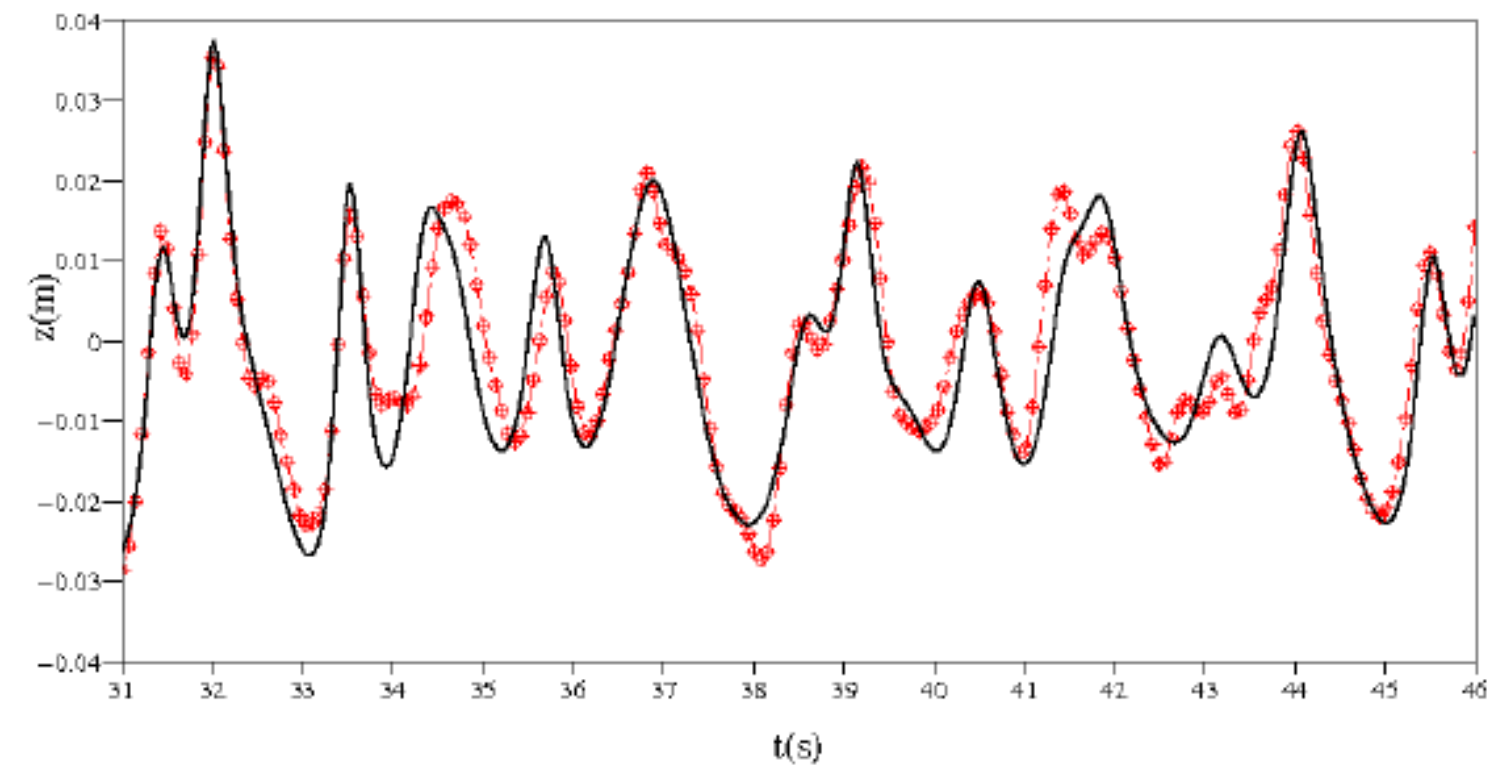

Figure 7. Profils de la surface libre à la sonde 16 pour le cas 26 de BECQ-GIRARD et al. (1999) : résultats du modèle en noir, résultats expérimentaux en rouge.

\section{Conclusions et perspectives de développement}

Le modèle présenté ici possède de nombreux avantages. D'un point de vue mathématique et numérique, il n'est composé que de 4 équations en une ou deux dimensions d'espace horizontales, ne comporte que des dérivées spatiales d'ordre deux au maximum et concentre l'essentiel de sa complexité dans un opérateur statique (i.e. qui peut être calculé une fois pour toute en début de simulation). Il possède de plus des propriétés linéaires dispersives extrêmement étendues, jusqu'en eaux profondes. Les 
validations présentées ici sur deux cas expérimentaux confirment les très bonnes capacités du modèle au niveau dispersif, mais également au niveau des interactions nonlinéaires. Les résultats obtenus sur ces cas-tests exigeants sont de très bonne facture, et supérieurs à ceux de modèles de type Boussinesq étendu, tels que NWOGU (1993) ou WEI et al. (1995) par exemple.

Les travaux actuels et ultérieurs concernent la réalisation d'autres cas-tests de validation (cas d'onde solitaire impactant un mur, instabilités de modulation de Benjamin-Feir), avant d'étendre le modèle aux cas de situations 2DH en utilisant des maillages nonstructurés. Au plan physique, on envisage également d'ajouter la prise en compte de processus dissipatifs d'énergie, déferlement et frottement sur le fond notamment.

\section{Références bibliographiques}

AGNON Y., MADSEN P.A., SCHÄFFER H.A. (1999). A new approach to high-order Boussinesq models. J. Fluid Mech., 399, pp 319-333. doi:10.1017/S0022112099006394

BECQ-GIRARD F., FORGET P., BENOIT M. (1999). Non-linear propagation of unidirectional wave fields over varying topography. Coastal Eng., 38, pp 91-113. doi:10.1016/S0378-3839(99)00043-5

BINGHAM H.B., AGNON Y. (2005). A Fourier-Boussinesq method for nonlinear water waves. Eur. J. Mech. B/Fluids, 24, 255-274. doi:10.1016/j.euromechflu.2004.06.006

CHAZEL F., BENOIT M., ERN A., PIPERNO S. (2009). A double-layer Boussinesqtype model for highly nonlinear and dispersive waves. Proc. R. Soc. Lond., A 465, pp 2319-2346. doi:10.1098/rspa.2008.0508

DINGEMANS M. (1994). Comparison of computations with Boussinesq-like models and laboratory measurements. Mast-G8M note, H1684. Delft Hydraulics, Pays-Bas.

GOBBI M.F., KIRBY J.T., WEI G. (2000). A fully nonlinear Boussinesq model for surface waves. Part 2. Extension to $O\left((k h)^{4}\right)$. J. Fluid Mech., 405, pp 181-210. doi:10.1017/S0022112099007247

LYNETT P., LIU P.L.-F. (2004). A two-layer approach to wave modeling. Proc. R. Soc. Lond., A 460, pp 2637-2669. doi:10.1098/rspa.2004.1305

MADSEN P.A., BINGHAM H.B., LIU H. (2002) A new Boussinesq method for fully nonlinear waves from shallow to deep water. J. Fluid Mech., 462, pp 1-30. doi:10.1017/S0022112002008467

NWOGU O.G. (1993). An alternative form of the Boussinesq equations for nearshore wave propagation. J. Waterway, Port, Coastal and Ocean Eng., 119(6), pp 618-638. doi:10.1061/(ASCE)0733-950X(1993)119:6(618)

PEREGRINE D.H. (1967). Long waves on a beach. J. Fluid Mech., 27, pp 815-827. doi:10.1017/S0022112067002605

WEI G., KIRBY J.T., GRILLI S.T., SUBRAMANYA R. (1995). A fully nonlinear Boussinesq model for surface waves: Part I. Highly nonlinear unsteady waves. J. Fluid Mech., 294, pp 71-92. doi:10.1017/S0022112095002813 